\title{
ABDOME AGUDO OCLUSIVO POR HEMATOMA DE INTESTINO DELGADO: RELATO DE CASO
}

\section{SPONTANEOUS INTRAMURAL SMALL-BOWEL HEMATOMA CAUSING OCCLUSIVE ACUTE ABDOMEN: CASE REPORT.}

Hector Sbaraini Fontes1; Fabiola Grigoreto Lupion1; Emanoela Rodrigues Azevedo1; Lydio Barbier Neto1; Sérgio Ossamu Ioshii2; Benur Polonio3

\section{RESUMO}

OBJETIVO: Relatar o caso de uma paciente adulta com abdome agudo oclusivo causado por hematoma de intestino delgado. MÉTODO: Apresenta-se o caso de uma paciente atendida pelo serviço de Cirurgia de Urgência e Emergência do HC-UFPR. RESULTADO: Paciente feminina, de 20 anos, com história de anemia aplásica, apresentou dor no mesogástrio, distensão abdominal e vômitos. A tomografia abdominal evidenciou segmento de intestino delgado com espessamento de sua parede. Foi submetida a laparotomia mediana onde se evidenciou um hematoma em alça ileal a $40 \mathrm{~cm}$ da válvula ileocecal, causando obstrução. Realizou-se enterectomia segmentar de $15 \mathrm{~cm}$ e enteroenteroanastomose. 0 exame anatomopatológico evidenciou hematoma extenso de submucosa do segmento ressecado. CONCLUSÃo: Os casos de abdome agudo oclusivo por hematoma de intestino delgado espontâneo são raros e devem ser manejados com tratamento clínico. Indica-se a cirurgia apenas na falha deste ou em casos de complicações durante o tratamento clinico.

Descritores: abdome agudo, intestino delgado, anemia aplásica.

\section{ABSTRACT}

BACKGROUND: We present a case report of a 20-year-old female with occlusive acute abdomen secondary to spontaneous intramural small-bowel hematoma. METHODS: Tomography demonstrate wall and mucosal thickening and "stack-of-coins appearance". During the laparotomy a $30 \mathrm{~cm}$ small bowel segment was resected. Histology confirmed submucosal layer hemorrhage. CONCLUSION: Spontaneous intramural small-bowel hematoma is rare. The incidence is expected to increase. This is because of several factors, including a growing number of patients with hematologic malignancies receiving chemotherapy and an aging population requiring more long-term anticoagulant therapy. It shoud usually be managed nonoperatively. Computed tomography is the imaging technique of choice. An operation shoud be advised if the small-bowel abnormality might be malignant, if the diagnosis is uncertain, or if there is active intraluminal hemorrhage, perforation, or ischemia.

Keywords: hypothyroidism, myxedema ascites and ascites.

\footnotetext{
1- Médico Residente de Cirurgia Geral, HC-UFPR.

2- Professor Associado de Patologia, UFPR e PUCPR.

3- Professor Adjunto de Técnica Cirúrgica e Cirurgia Experimental, UFPR.
} 


\section{INTRODUÇÃO}

As causas mais frequentes de abdome agudo oclusivo são aderências, hérnias e neoplasia. As outras patologias são afecções raras, que representam menos de $10 \%$ dos casos. Dentre estas, está o hematoma de intestino delgado ${ }^{8}$. Existem relatos de casos na literatura, sendo a anticoagulação com warfarina considerada a principal etiologia, com uma incidência de 1:25.000 pacientes tratados com anticoagulantes por ano ${ }^{10}$. Contudo pode estar associada a leucemia, linfoma, mieloma, hemofilia, quimioterapia, pancreatite, carcinoma de pâncreas, poliarterite nodosa, púrpura de HenochShonlein, doença de Von Willebrand e púrpura trombocitopenica idiopática1,3. É mais frequente no jejuno, mas pode ser achado em qualquer ponto do trato gastrointestinal. 0 quadro clínico mais frequente é a dor abdominal, normalmente associada com outras manifestações hemorrágicas. A oclusão intestinal pode estar presente. 0 diagnóstico pode ser feito através de exames de imagem, especialmente a tomografia computadorizada. O tratamento é clínico em casos não complicados, com ajuste da anticoagulação, tratamento para a doença de base e manejo da suboclusão intestinal. Nos pacientes com falha do tratamento clínico ou com complicações, está indicado tratamento cirúrgico ${ }^{1,8}$.

\section{RELATO DE CASO}

Paciente de 20 anos, feminina, com anemia aplásica severa em uso de imunossupressores para realização de transplante de medula óssea no HC-UFPR. Internada no serviço por quadro de dor abdominal tipo cólica há 4 dias, difusa e contínua com períodos de piora, associado a náusea e vômitos e parada de evacuações, sendo solicitada a avaliação da equipe de Cirurgia Geral do serviço de Urgência e Emergência. Apresentava distensão abdominal e dor difusa à palpação superficial. A tomografia computadorizada com contraste endovenoso evidenciou distensão gástrica e de alças de intestino delgado que se estendiam até ponto de transição na topografia da junção jejuno-ileal, com alças colabadas a jusante (Figura 1).

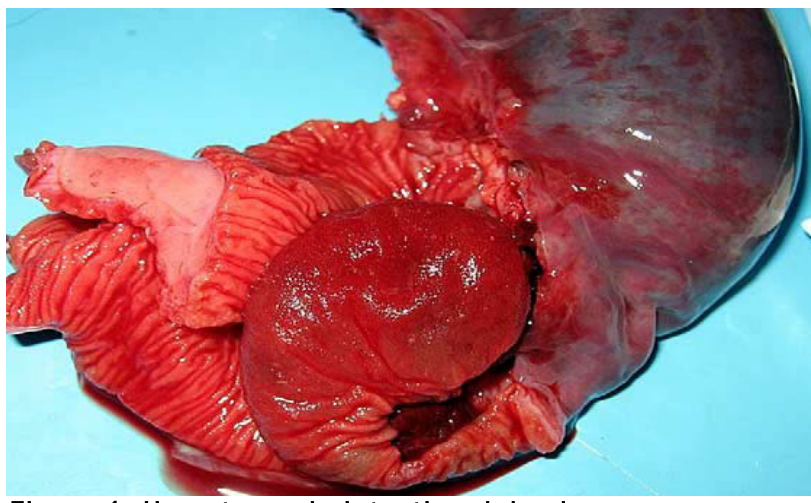

Figura 1: Hematoma de intestino delgado.
No local, havia uma imagem heterogênea na parede da alça, podendo corresponder a um hematoma intramural. Foi então realizada sondagem nasogástrica com saída imediata de $3000 \mathrm{ml}$ de estase e a paciente evoluiu sem melhora da dor, mantendo o volume alto da drenagem pela sonda. Indicada videolaparoscopia diagnóstica que evidenciou segmento entérico com hematoma de parede com aproximadamente $30 \mathrm{~cm}$ de extensão não se podendo descartar isquemia. Realizada conversão para laparotomia mediana pela impossibilidade de tratamento por via laparoscópica. O achado foi um segmento de íleo localizado a $40 \mathrm{~cm}$ da válvula ileocal com extenso hematoma em sua parede (Figura 2). Realizou-se a ressecção do segmento acometido e enteroenteroanastomose. 0 exame anatomopatológico evidenciou hematoma extenso de submucosa e infarto mucoso de intestino delgado. A paciente evoluiu com

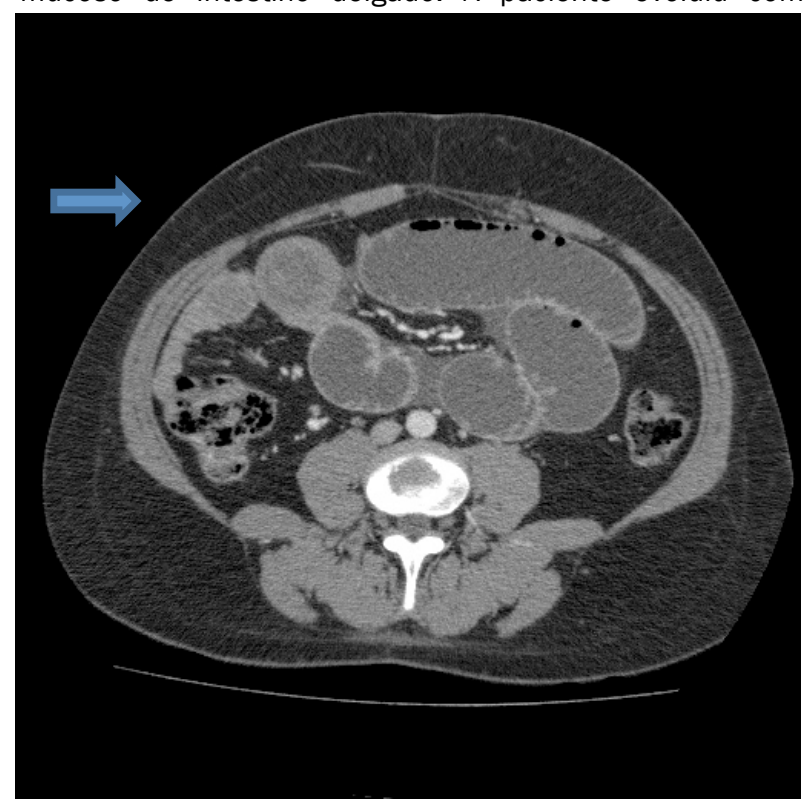

melhora progressiva, recebendo alta.

Figura 2: Tomografia computadorizada de abdome mostrando espessamento da parede intestino delgado e dilatação proximal.

\section{DISCUSSÃO}

O abdome agudo oclusivo por hematoma intramural espontâneo de intestino delgado é uma condição rara, usualmente associada a anticoagulação. Outros fatores de risco associados com esta afecção seriam: leucemia, linfoma, mieloma, hemofilia, quimioterapia, pancreatite, câncer de pâncreas, poliarterite nodosa, púrpura de Henoch-Schonlein, púrpura trombocitopenica idiopática e ingestão de algumas substâncias como veneno para ratos 1,10 . Sutherland em 1904, descreveu o primeiro caso de hematoma não traumático de intestino delgado causando abdome agudo obstrutivo por intussucepção em uma criança de 5 anos portadora de púrpura de Henoch-Schonlein4.

Usualmente os pacientes apresentam dor abdominal associada ao uso de anticoagulantes. Contudo 
pode ocorrer em pacientes com alteração da coagulação por outras patologias como anemias e leucemias, como no caso acima descrito.

A ecografia abdominal pode auxiliar no diagnóstico, contudo o exame de eleição é a tomografia abdominal, com uma sensibilidade próxima de $100 \%$. Ela mostra espessamento da parede intestinal (imagem de pseudorim) e o nível de sua obstrução2,5,7,9, achado este presente na TC da paciente. 0 jejuno é o segmento mais acometido, seguido pelo duodeno e íleo.

O tratamento deve ser inicialmente conservador, como o realizado, com suspensão ou ajuste da anticoagulação (com o uso de plasma fresco e vitamina $\mathrm{K}$ ) e descompressão com sonda nasogástrica, haja visto que a maioria dos casos melhora sem cirurgia7. Apenas na falha do tratamento clinico ou na presença de complicações como isquemia e perfuração o tratamento cirúrgico é indicado $3,6,8$.

Após a melhora do quadro clínico, a anticoagulação pode ser reiniciada, não estando necessariamente associada a novos episódios de hematomas ${ }^{1}$. A recorrência de sangramento assim como sequelas tardias como estenoses são raras ${ }^{8}$.

\section{CONCLUSÃO}

O abdome agudo obstrutivo causado por hematoma de intestino delgado é raro, mas apresenta especial importância em um pequeno grupo de pacientes. Esta etiologia é comum em paciente em uso de anticoagulantes, mas também pode estar presente em portadores de doenças hematológicas. O manejo é predominantemente clínico, mas o tratamento cirúrgico tem sua indicação na falha do primeiro e na presença de complicações.

\section{REFERÊNCIAS}

1. Abbas MA, collins JM, olden KW, kelly $K A$ Spontaneous intramural small-bowel hematoma. Clinical presentation and long-term outcome. Arch Surg. 2002;137:306-10.

2. Çarkman S, Ozben V, Saribeyoglu K, Somuncu E, Erguney S, Korman U, Pekmezci S. Spontaneous intramural hematoma of the intestine. Ulus Trauma derg. 2010;16(2):165-9.

3. Delibegovic M, Alispahic A, Gazija J, Mehmedovic Z, Mehmedovic M. Intramural haemorrhage and haematoma as the cause of ileus of the small intestine in a haemophiliac. Med Arch. 2015;69(3):206-7.

4. Hughes CE, Conn J, Sherman JO. Intramural hematoma of the gastrointestinal tract. Am J Surg. 1977;133:276-9.

5. Lin TP, Liu CH. Spontaneous intramural hematoma of the small intestine. Intern Med. 2014;53(22):2647.

6. Kalu ON, Al-khoury G, Reck CA, Velcek F. Submucosal hematoma presenting as small bowel obturator obstruction in a patient on low-molecular-weight heparin. J Ped Surg. 2008;43:1569-71.
7. Polat C, Dervisoglu A, Guven H, Kaya E, Malazgirt Z, Danaci M, Ozkan K. Anticoagulant-induced intramural intestinal hematoma. Am J Emerg Med. 2003;21;208-11.

8. Sorbello MP, Utiyama EM, Parreiro JG, Birolini D, Rasslan S. Spontaneous intramural small bowel hematoma induced by anticoagulant therapy: review and case report. Clinics. 2007;62(6);785-90.

9. Yoldas T, Erol V, Çaliskan C, Akgun E, Korkut M. Spontaneous intestinal intramural hematoma: what to do and not to do. Ulus Cerrahi Derg. 2013;29(2):72-5.

10. Zammit A, Marguerat DG, Caruana C. Anticoagulationinduced spontaneous intramural small bowel haematomas. BMJ. 2013; jun;1-3. 